\title{
Perspectivas geocientíficas del enriquecimiento supergénico de atacama y prospección geológica en desiertos meridionales del Perú
}

\author{
Bachiller Mario Dominico Rodríguez Delgado \\ mario.rodriguez8@unmsm.edu.pe \\ Alumno de la Unidad de Posgrado. \\ Facultad de Ingeniería Geológica, Minera, Metalúrgica y Geográfica. \\ Universidad Nacional Mayor de San Marcos. \\ Dr. Jaime Cesar Mayorga Rojas \\ jaime.mayorga@unmsm.edu.pe \\ Docente de la unidad de Posgrado. \\ Facultad de Ingeniería Geológica, Minera, Metalúrgica y Geográfica. \\ Universidad Nacional Mayor de San Marcos \\ Unidad de Posgrado. Av. Venezuela cuadra $34 \mathrm{~s} / \mathrm{n}$, \\ Ciudad Universitaria, Lima, Perú.
}

\section{RESUMEN}

La explotación de yacimientos Porfiríticos son importantes contribuyentes a la humanidad con metales como el $\mathrm{Au}, \mathrm{Cu}$ y Mo y se constituyen como una de las mayores actividades extractivas en el mundo, ahora su descubrimiento y puesta en valor nos dirige a tener como objetivo la investigación en entornos tectónicos de subducción del cinturón del pacífico cada vez más complejos y bajo gruesas capas transportadas de cubierta pre o post mineral, para ello las herramientas conceptuales indirectas juegan un rol importante para el éxito en perspectivas futuras. En este artículo se exponen modernas perspectivas de investigación; como un breve análisis de las fluctuaciones climáticas acaecidas en el neógeno, la metodología geoquímica de microfases y el uso de trazadores isotópicos como herramientas geocientíficas para la comprensión de la mineralización supergénica del desierto de Atacama para así plantear una metodología prospectiva de ubicación de la fuente de los metales e improntas geoquímicas de contraste en otros ambientes tectónicos similares como los desiertos de la costa sur del Perú.

Palabras clave: perspectivas geocientificas; enriquecimiento supergénico; desierto de atacama; fluctuaciones climáticas, trazadores isotópicos. 
Rodríguez Delgado y Mayorga Rojas...

\title{
Geoscientific perspectives on atacama supergene enrichment and geological prospecting in southern Peruvian deserts
}

\begin{abstract}
The exploitation of porphyritic deposits are important contributors to humanity with metals such as $\mathrm{Au}, \mathrm{Cu}$ and $\mathrm{Mo}$ and constitute one of the largest extractive activities in the world, now their discovery and valorization is leading us to focus on research in increasingly complex subduction tectonic environments of the Pacific belt and under thick transported layers of pre- or post-mineral cover, for which indirect conceptual tools play an important role for the success of future perspectives. This paper presents modern research perspectives, such as a brief analysis of Neogene climate fluctuations, microphase geochemical methodology and the use of isotopic tracers as geoscientific tools for understanding the supergene mineralization of the Atacama Desert in order to propose a prospective methodology for metal source localization and contrasting geochemical imprints in other similar tectonic environments such as the deserts of the southern Peruvian coast.
\end{abstract}

Key words: geoscientific perspectives; supergene enrichment; atacama desert; climatic fluctuations, isotopic tracers.

Artículo recibido: 02 noviembre. 2021 Aceptado para publicación: 28 noviembre 2021 Correspondencia: mario.rodriguez8@unmsm.edu.pe Conflictos de Interés: Ninguna que declarar 


\section{INTRODUCCIÓN}

La mineralización supergénica de los depósitos de cobre del desierto de Atacama acaecida en el Cenozoico, ha desempeñado un papel fundamental en la génesis de esta importante provincia minera, la mayor productora de cobre en el mundo, en ella, fluctuaciones climáticas han desencadenado, como epilogo; una profunda hiperaridez, este proceso climático generado por condiciones meteorológicas similares al fenómeno del niño (figura1), donde la subsidencia atmosférica subtropical, la inversión de temperaturas debido al afloramiento costero de la corriente fría Perú-Chile o corriente de Humboldt, además de un efecto continental de distancia al atlántico y un efecto de sombra de lluvia en el flanco oriental de los andes que recolectan la escasa humedad proveniente de la zona tropical del Brasil, unido a altas tasas de evaporación intensificaron las condiciones hiperaridas del desierto. (Benedikt Ritter, et, al 2018). Después de la fase principal de enriquecimiento supergénico oxidativo, se depositó un gran volumen de sedimentos clásticos procedente de la Pre cordillera en la Depresión Central, mientras que simultáneamente otros depósitos quedaban expuestos, lo que permitió, iniciar la explotación minera en Chuquicamata en la época preincaica.

En la actualidad las exploraciones se centran en posibles depósitos ocultos bajo estas densas capas de material cuaternario y los estudios realizadas inicialmente en 1999 en mina Radomiro Tomic, sugirieron que las anomalías son expresión superficial de la modificación del conjunto supergénico primario por fluidos ascendentes salinos; que trepan favorecidos por controles estructurales y por bombeo sísmico, en el ascenso ganan metales y modifican el conjunto supergénico a uno que contiene atacamita. (Eion M. Cameron et al.2010).

Numerosos estudios han documentado datos geoquímicos de anomalías en la sobrecarga transportada por encima de los depósitos minerales enterrados en muchos entornos diferentes. En estos estudios se han aplicado una variedad de enfoques, incluida la lixiviación selectiva de suelos para extraer el componente móvil de los elementos (Cameron et al. 1998). Las anomalías geoquímicas alojadas en las gravas sobre los depósitos de pórfidos de cobre enterrados en el desierto de Atacama, como Spence (Cameron \& Leybourne 2005), Mantos Blancos (Palacios et al. 2005), Radomiro Tomic, Mansa Mina (Cameron et al. 2010), y Gaby Sur (Cameron et al. 2004), se han 
caracterizado por elevadas concentraciones de sal $(\mathrm{NaCl})$ y elementos indicadores de pórfidos de cobre (Cu, Mo, Re, Se, As).

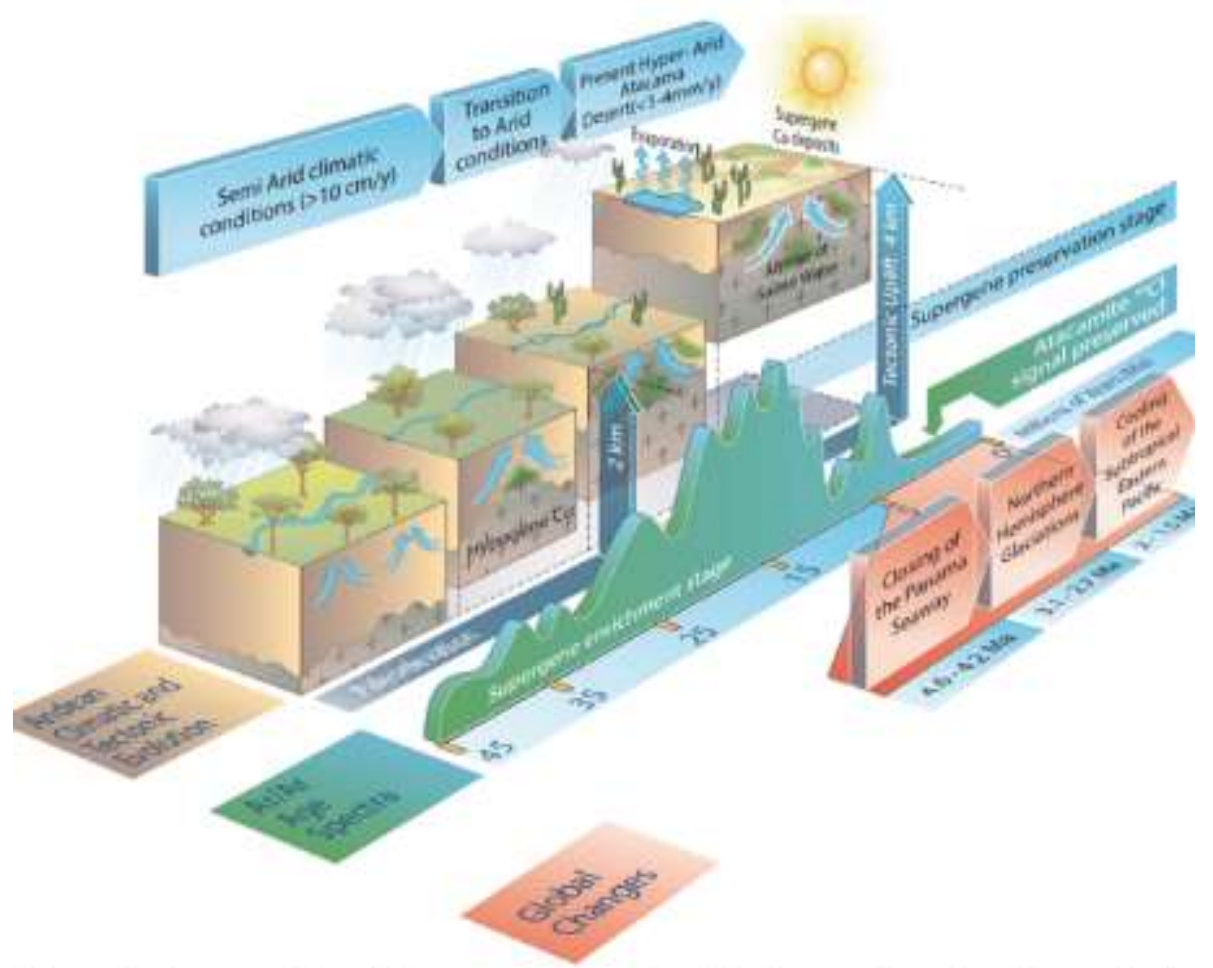

Representación esquemática de los procesos de enriquecimiento supergenico durante la evolución climática y tectónica de los Andes. La oxidación y el enriquecimiento supergénico prolongado se producen en un escenatio de cambio climático caracterizado por la transición de un clima hưmedo en condiciones semiaridas (tasas de precipitación $>10 \mathrm{~cm} / \mathrm{a}$ ) a condiciones más secas y áridas. Bajo el clima hiperárido actual (precipitación $<1-4 \mathrm{~mm} / \mathrm{a}$ ), los conjuntos de cobre supergenico son modificados por las aguas salinas ascendentes, dejando firmas de ${ }^{\circ}{ }^{\circ} \mathrm{Cl}$ que se conservan debido a la falta de lluvias. Se muestra el espectro de edad ${ }^{40} \mathrm{Ar} / \mathrm{Ar}$ para los últimos $45 \mathrm{Ma}$. La escala vertical indica la variación de las condiciones de humedad, alcanzando un pico en -20-15 Ma en el desierto de Atacama. Se destacan los cambios globales que afectan al clima en los últimos $5 \mathrm{Ma}$. (Paulo M. Vasconcelos et al. 2015)

La formación de anomalías geoquímicas ha sido interpretada por Cameron et al. (2002) como el resultado de la lixiviación de elementos solubles y móviles en la interacción de las aguas subterráneas con la mineralización, seguida del transporte de estas aguas enriquecidas en oligoelementos (figura 2) a la superficie por bombeo inducido por la alta actividad sísmica y a través de zonas de fractura que se extienden en las gravas suprayacentes.

El propósito fundamental de esta investigación es la comprensión de estas metodologías de prospección geológica en zonas de mineralización supergénica del desierto de Atacama a fin de poder desarrollar una perspectiva acorde a nuestra realidad basada en estas tecnologías y su posterior aplicación, destacando la importancia central de estos 
estudios, pues permitiría sentar las bases conceptuales de la prospección geológica de depósitos minerales en los desiertos de los departamentos de Tacna y Moquegua

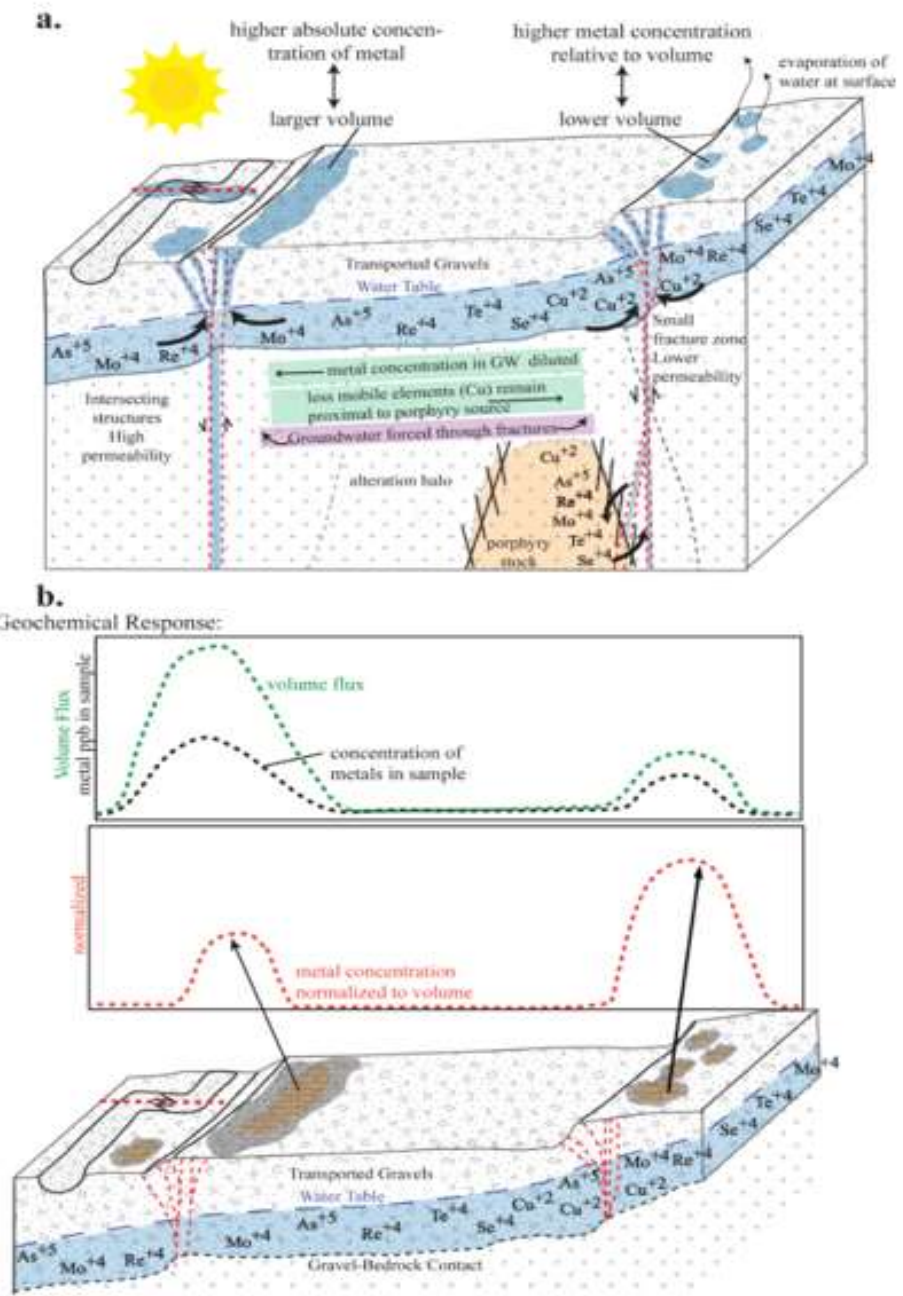

Figura 2. Modelos conceptuales (a) transporte de oligoelementos a la superficie y volúmenes variables de flujo de aguas subterráneas a la superficie; (b) normalización de la firma de metales para el flujo de aguas subterráneas y principales cationes y aniones en las aguas subterráneas (Alexandra Brown et al, 2019)

Resaltados por sus similitudes Paleoclimáticas, geotectónicas, sísmicas y petrológicas con el norte de Chile. La aplicación de los isótopos de los metales de transición, como el $\mathrm{Cu}$, Mo y $\mathrm{Zn}$, es una herramienta nueva, pero a la vez muy potente en hidrogeoquímica. En las dos últimas décadas, los avances en las técnicas analíticas de MC ICP-MS han permitido realizar mediciones muy precisas de los isotopos de los metales de transición de entre +/- 0,1 y 0,2\%o. la aplicación de isótopos estables e isótopos radiogénicos es un enfoque prometedor para rastrear la fuente de los elementos indirectos, así como sus 
mecanismos de liberación; aunque los llamados isótopos estables ligeros (H, C, O y S) se utilizaron en este contexto durante décadas. El significativo contraste en la movilidad del $\mathrm{Cu}$ y el Mo a través de la química de las aguas subterráneas ofrece una oportunidad para identificar anomalías y tendencias en las aguas subterráneas con un amplio espacio de muestra y un vector que se remonta a la mineralización. Sus mecanismos de dispersión se ilustran en la Figura 3.

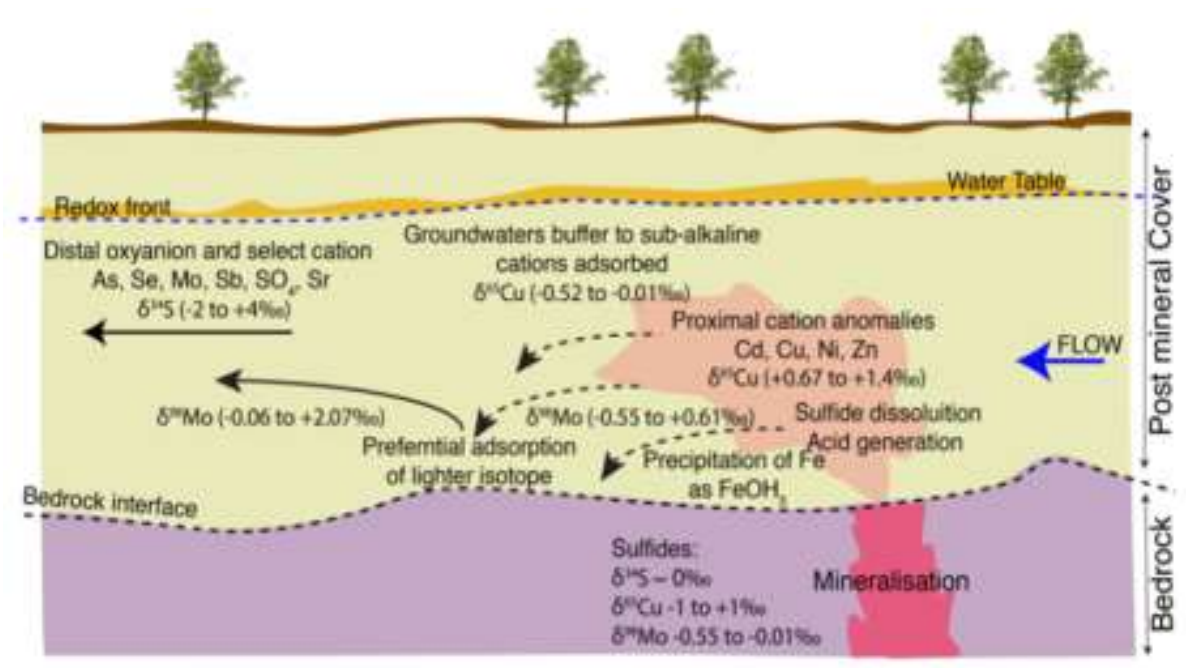

Modelo conceptual para las huellas geoquimicas de trazas e isótopos en las aguas subterriaeas próximas y descendentes de los yacimientos minerales. (James Kidder et al. 2020).

\section{ESTRATEGIAS METODOLOGICAS}

El análisis de la información bibliográfica nos ha permitido construir mapas conceptuales, redes semánticas, lluvia de ideas, formulación de hipótesis, construcción de gráficos y cuadros que finalmente nos han facilitado identificar principios, criterios y técnicas utilizadas en la prospección geológica de anomalías geoquímicas y paleoclimáticas en desiertos a partir de la información relacionada al enriquecimiento supergénico en el desierto de atacama y de un clúster de yacimientos minerales en el norte de chile que comparten una génesis similar por haber acaecido los eventos mineralizadores de manera simultánea.

El criterio que ha primado para la selección de la información bibliográfica está relacionado hacia el análisis de las nuevas metodologías y tecnologías de prospección geológica en entornos desérticos y cubiertos por gruesas capas de material aluvial, analizando artículos científicos de estudios realizados principalmente en el norte de Chile. 


\section{RESULTADOS}

\subsection{Fluctuaciones Climáticas}

\subsubsection{Evolución paleoclimática global.}

Muchos estudios geocronológicos ${ }^{40} \mathrm{Ar} /{ }^{39} \mathrm{Ar}$ de depósitos de Mn en África (Beauvais et al. 2008), Australia (Dammer et al. 1999; Li y Vasconcelos 2002; Vasconcelos 2002; Feng y Vasconcelos 2007; Vasconcelos et al. 2013), China (Li et al. 2007; Deng et al. 2014) India (Bonnet et al. 2014), y Europa (Hautmann y Lippolt 2000) revelan ahora historias comparables, en las que la meteorización y las precipitaciones minerales intermitentes a lo largo de todo el Cenozoico sugieren la alternancia de períodos húmedos y secos que, a su vez, reflejan las condiciones climáticas globales (Figura 4). Hautmann y Lippolt 2000) prefieren atribuir esta historia de precipitación mineral intermitente a los controles tectónicos, pero no se puede identificar claramente un mecanismo que pueda relacionar las fuerzas tectónicas con la precipitación mineral en la corteza meteorizada.

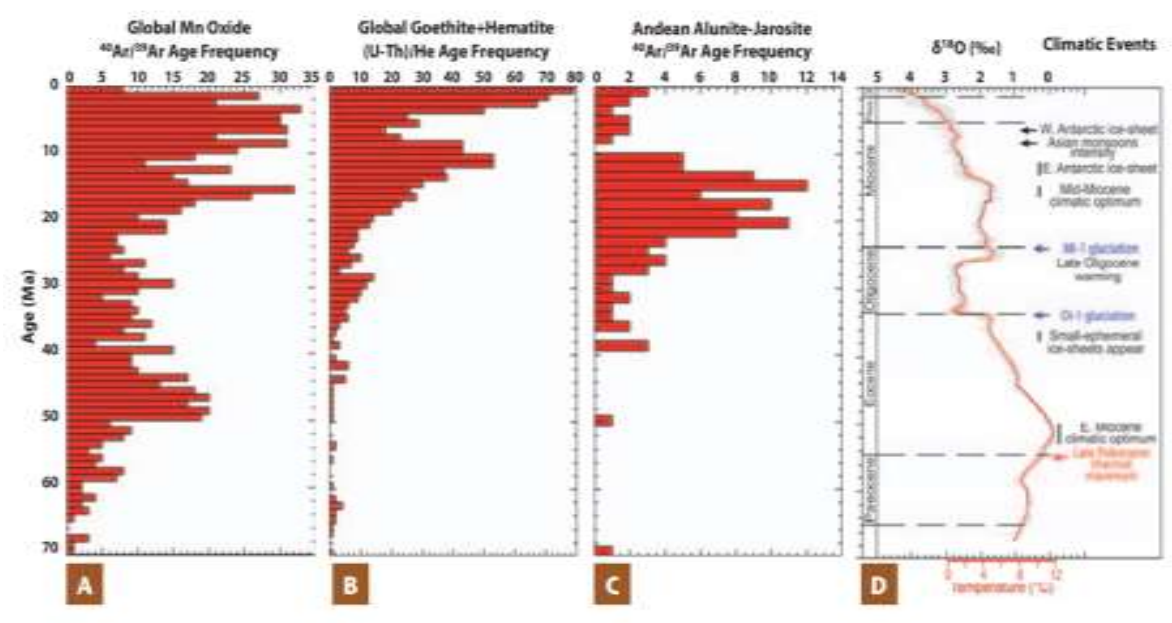

Figura 4. (A) Histograma de distribución global de edades de los óxidos de Mn supergénico. (B) Histograma de distribución global de edades de las goethitas + hematitas supergénicas. (C) Histograma de distribución de edades de la alunita-jarosita supergénica andina. La distribución de los minerales supergénicos a través del tiempo ayuda a identificar periodos del pasado geológico propicios para la disolución y reprecipitación de los elementos de mena en entomos de meteorización. Los óxidos de Mn registran condiciones húmedas. Por el contrario, la formación y preservación de alunita-jarosita supergénica requiere condiciones, originadas por el descenso del nivel freático durante una transición de condiciones húmedas o semiáridas a hiperáridas. (D) Gráfico de los cambios de temperatura global desde el Paleoceno hasta el Reciente. Este gráfico ayuda a explicar las condiciones de meteorización reflejadas en las figuras de A a C. (Zachos et al. 2001).

\subsubsection{Registros paleoclimáticos regionales}

La abundancia y la estabilidad relativa de los óxidos de K-Mn en las lateritas de Mn conservan un amplio registro de la meteorización. La hollandita $\left(\mathrm{Ba}\left(\mathrm{Mn}_{6}{ }^{4+} \mathrm{Mn}_{2}{ }^{3+}\right) \mathrm{O}_{16}\right)$ y el criptomelano $\left(\mathrm{K}\left(\mathrm{Mn}_{7}^{4+} \mathrm{Mn}^{3+}\right) \mathrm{O}_{16}\right)$, principales minerales de las lateritas de $\mathrm{Mn}$, 
proporcionan una historia detallada de la meteorización y de la evolución paleoclimática continental (Vasconcelos 1999b) cuando se datan por el método ${ }^{40} \mathrm{Ar} /{ }^{39} \mathrm{Ar}$. Por ejemplo, En el distrito de Carajás (Brasil), la geocronología de los óxidos de K-Mn reveló una historia de precipitaciones intermitentes pero abundantes, a lo largo del Cenozoico.

El estudio de los depósitos supergénicos de Mn en otros lugares de Brasil (de Oliveira Carmo y Vasconcelos 2006; Spier et al. 2006) muestra una historia de meteorización igualmente prolongada, que comienza en $\sim 67$ Ma y continúa hasta el presente. Esta historia es intermitente, lo que revela una estrecha relación entre la evolución de los perfiles de meteorización y las condiciones climáticas globales.

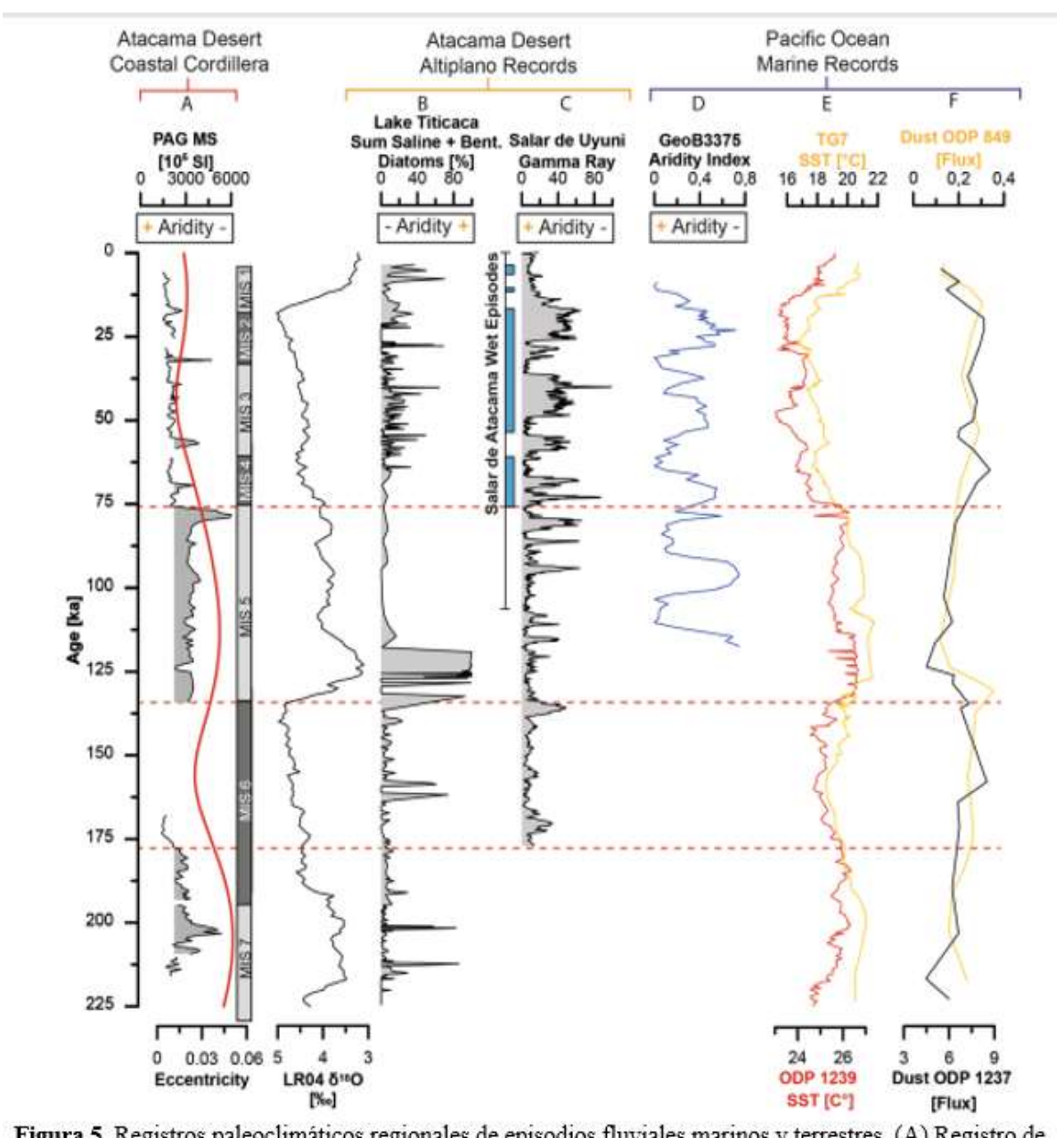
susceptibilidad magnética y línea roja de excentricidad local. (B) Pila global de isótopos de oxigeno en aguas profundas. (C) Gráfico de suma de especies de diatomeas salinas y bentónicas del lago Titicaca (D) Registro de rayos gamma del Salar de Uyuni y episodios húmedos del Salar de Atacama. (E) Índice de aridez $\left(27^{\circ}\right.$ S) reconstruido por Stuut y Lamy. (F) Reconstrucciones de TSM del Pacifico oriental a $17^{\circ} \mathrm{S}$, y del Pacifico ecuatorial. (G) Registros de flujo de polvo de $16^{\circ} \mathrm{S}$ y del Pacifico ecuatorial. (Benedikt Ritter et al. 2018) 
La reconstrucción paleoclimática de la Cordillera de la Costa indica una tendencia opuesta a la disponibilidad de humedad en comparación con los registros continentales de Atacama/Altiplano, que están influenciados principalmente por la advección de humedad desde las masas de aire del Atlántico y las precipitaciones en los altos Andes (Fig. 5). Esto muestra la separación de las fuentes de humedad que afectan a los sistemas de drenaje de la Cordillera de la Costa. La coincidencia con los registros paleoclimáticos del Pacífico marino, apunta a una conexión de episodios "más húmedos" en la Cordillera de la Costa con el aumento de las TSM de Chile/Perú durante los tiempos interglaciares (Fig. 5). La reconstrucción indica además que los periodos húmedos, caracterizados por una mayor actividad fluvial dentro de la cuenca que transporta gruesa arena a un lago efímero, ocurrieron predominantemente durante los tiempos interglaciares, mientras que los periodos secos, con una sedimentación atenuada de sedimentos finos, tuvieron lugar durante los tiempos glaciares. La alternancia glaciar-interglaciar durante el MIS 7 y el MIS 5 imita un control del ritmo de la excentricidad sobre la humedad en la Cordillera de la Costa (Fig. 5). (Benedikt Ritter, et, al 2018).

\subsection{Los nuevos métodos analíticos de microfases}

La geoquímica de exploración tradicional ha centrado sus esfuerzos en estudiar los diversos tipos de halos y trenes de dispersión elemental secundaria y halos de dispersión primaria expuestos en la superficie terrestre. Ha obtenido muchos éxitos en el descubrimiento de cuerpos minerales. Pero es mucho menos eficiente para descubrir cuerpos minerales ocultos profundamente bajo varios tipos de sobrecargas transportadas. Por tanto, se está prestando cada vez más atención al desarrollo de la geoquímica de penetración profunda. Numerosos estudios de casos publicados han documentado datos geoquímicos superpuestos de cubiertas de mineral bajo 50 a 100 m de grava piamontesa. Por lo tanto, es imposible utilizar métodos tradicionales de exploración geoquímica de superficie para detectar depósitos ocultos debajo de una cubierta exógena tan gruesa. Los llamados métodos de "análisis de fases" en muestras de mineral se desarrollaron originalmente en Rusia. En China, estos métodos se han desarrollado aún más en muestras de suelo anómalas con el objetivo de evaluar la importancia de las anomalías metálicas (Gong, 1994, 2007). En este artículo, se expone los métodos de análisis de fase modificados para determinar las fases oxidadas y sulfuradas de $\mathrm{Cu}$ en muestras de suelo recolectadas en sobrecargas exógenas. Debido a que la concentración de elementos en 
estas fases está en el rango de ppm y sub-ppm, los métodos analíticos descritos a continuación se denominan más apropiadamente análisis de microfases. (Xuejing Xie, et al. 2011). Estos métodos se usaron para analizar dos tipos de muestras de suelo con anomalías geoquímicas con el objetivo de evaluar la significación de estas anomalías metálicas, muestras de fracción de malla 80 y muestras procesadas de tamaño de malla 120, a fin de investigar si las muestras de grano más fino conducirían a resultados anómalos consistentes y sus resultados se muestran a continuación en las tablas 1,2 y 3 también en la figura 5.

Tabla 1 datos del Cobre de la fase oxidada a lo largo de la línea 7480500. (Xuejing Xie, et al. 2011)

\begin{tabular}{|c|c|c|c|c|c|c|c|c|}
\hline \multirow[t]{2}{*}{$\mathrm{N}^{\mathrm{o}}$} & \multicolumn{2}{|c|}{$\begin{array}{c}\text { Fase oxidada de } \mathrm{Cu} \\
(\mathrm{ppm})\end{array}$} & \multirow[t]{2}{*}{$\mathrm{N}^{\circ}$} & \multicolumn{2}{|c|}{$\begin{array}{c}\text { Fase oxidada de } \mathrm{Cu} \\
(\mathrm{ppm})\end{array}$} & \multirow[t]{2}{*}{$\mathrm{N}^{\mathrm{o}}$} & \multicolumn{2}{|c|}{$\begin{array}{c}\text { Fase oxidada de } \mathrm{Cu} \\
(\mathrm{ppm})\end{array}$} \\
\hline & Malla 80 & Procesada & & Malla 80 & Procesada & & Malla 80 & Procesada \\
\hline 473549 & 15.8 & 18.2 & 474557 & 25 & 25 & 475120 & 12.7 & 14.6 \\
\hline 473595 & 16.8 & 17.2 & 474586 & 22.7 & 22.7 & 475279 & 18.3 & 18.3 \\
\hline 473650 & 18.8 & 16.7 & 474615 & 25 & 25 & 475316 & 15.8 & 16.7 \\
\hline 473693 & 16.7 & 15 & 474640 & 21.7 & 21.7 & 475367 & 17.2 & 16.7 \\
\hline 473745 & 18.7 & 16.5 & 474665 & 16.7 & 19.9 & 475412 & 16.7 & 16.5 \\
\hline 473792 & 16.7 & 16.5 & 474698 & 25 & 25 & 475463 & 15.8 & 15.8 \\
\hline 473840 & 16.7 & 16.5 & 474723 & 29.2 & 29.2 & 475511 & 16.7 & 16.8 \\
\hline 473890 & 18.4 & 16.7 & 474746 & 21.8 & 21.8 & 475564 & 16.7 & 17.6 \\
\hline 473940 & 15.8 & 15 & 474771 & 26.7 & 26.7 & 475611 & 12.5 & 16.5 \\
\hline 473989 & 16.8 & 17.2 & 474791 & 29.5 & 29.5 & 475659 & 14.8 & 16.2 \\
\hline 474039 & 16.7 & 16 & 474810 & 29.5 & 29.5 & 475709 & 15 & 15 \\
\hline 474087 & 15.8 & 16.7 & 474826 & 26.7 & 26.7 & 475758 & 16.7 & 16.7 \\
\hline 474134 & 16.7 & 16.7 & 474855 & 29.2 & 29.2 & 475808 & 15.8 & 15.8 \\
\hline 474184 & 16.7 & 17.2 & 474876 & 26.7 & 26.7 & 475857 & 20 & 20 \\
\hline 474233 & 15.8 & 16.7 & 474911 & 26.7 & 26.7 & 475907 & 15 & 15 \\
\hline 474286 & 17 & 16.7 & 474937 & 29.2 & 29.2 & 475951 & 15 & 15 \\
\hline 474319 & 16.2 & 16.2 & 474958 & 29.2 & 29.2 & 476004 & 16.7 & 16.5 \\
\hline 474380 & 15.8 & 16.7 & 474975 & 22.7 & 25 & 476049 & 20.8 & 20.8 \\
\hline 474425 & 16.7 & 17 & 475002 & 25 & 15.8 & 406097 & 21.7 & 21.7 \\
\hline 474473 & 16.7 & 20.8 & 475030 & 15.8 & 15.8 & Promedio & 19.55 & 19.64 \\
\hline 474521 & 22.8 & 22.8 & 475071 & 16.8 & 16.8 & & & \\
\hline
\end{tabular}


Tabla 2 datos de $\mathrm{Cu}$ de la fase de sulfuro a lo largo de la línea 7480500. (Xuejing Xie, et al. 2011)

\begin{tabular}{|c|c|c|c|c|c|c|c|c|}
\hline \multirow[t]{2}{*}{$\mathrm{N}^{\mathrm{o}}$} & \multicolumn{2}{|c|}{$\begin{array}{l}\text { Cu en fase de } \\
\text { sulfuro(ppm) }\end{array}$} & \multirow[t]{2}{*}{$\mathrm{N}^{\circ}$} & \multicolumn{2}{|c|}{$\begin{array}{c}\mathrm{Cu} \text { en fase de sulfuro } \\
(\mathrm{ppm})\end{array}$} & \multirow[t]{2}{*}{$\mathrm{N}^{\mathrm{o}}$} & \multicolumn{2}{|c|}{$\begin{array}{l}\text { Cu en fase de } \\
\text { sulfuro(ppm) }\end{array}$} \\
\hline & Malla 80 & Procesada & & Malla 80 & Procesada & & Malla 80 & Procesada \\
\hline 473549 & 7.1 & 10.5 & 474557 & 21.4 & 21.4 & 475120 & 21.4 & 17.9 \\
\hline 473595 & 11.2 & 20.8 & 474586 & 14.6 & 20.8 & 475279 & 10.7 & 10.7 \\
\hline 473650 & 7.1 & 14.2 & 474615 & 21.4 & 34 & 475316 & 10.7 & 14.2 \\
\hline 473693 & 8.4 & 15 & 474640 & 14.7 & 24 & 475367 & 17.9 & 17.9 \\
\hline 473745 & 7.1 & 14.2 & 474665 & 25 & 39.3 & 475412 & 17.9 & 16.7 \\
\hline 473792 & 15.4 & 14.5 & 474698 & 14.3 & 24.7 & 475463 & 17.9 & 17.1 \\
\hline 473840 & 15.9 & 15.2 & 474723 & 32.1 & 28.2 & 475511 & 14.2 & 17.5 \\
\hline 473890 & 14.2 & 15 & 474746 & 14.7 & 22 & 475564 & 14.2 & 14.2 \\
\hline 473940 & 7.1 & 14.6 & 474771 & 21.4 & 29.9 & 475611 & 10.7 & 10 \\
\hline 473989 & 13.9 & 16.2 & 474791 & 12.7 & 25 & 475659 & 17.9 & 13 \\
\hline 474039 & 14.2 & 14.2 & 474810 & 30.8 & 21.5 & 475709 & 21.4 & 21.4 \\
\hline 474087 & 14.4 & 14.4 & 474826 & 39.3 & 21.4 & 475758 & 17.9 & 21.4 \\
\hline 474134 & 10.7 & 14.2 & 474855 & 21.4 & 21.4 & 475808 & 17.9 & 21.4 \\
\hline 474184 & 8.6 & 17.9 & 474876 & 13.7 & 23.5 & 475857 & 14.2 & 21 \\
\hline 474233 & 14.6 & 17.9 & 474911 & 17.9 & 21.4 & 475907 & 14.2 & 17.9 \\
\hline 474286 & 10.7 & 10.7 & 474937 & 64.3 & 65 & 475951 & 17.9 & 14.2 \\
\hline 474319 & 21.4 & 10.7 & 474958 & 17.9 & 25 & 476004 & 14.2 & 32.1 \\
\hline 474380 & 10.7 & 21.4 & 474975 & 14.9 & 18.7 & 476049 & 44.3 & 20.8 \\
\hline 474425 & 14.2 & 21.4 & 475002 & 32.1 & 16.7 & 406097 & 15 & 15 \\
\hline 474473 & 21.4 & 14.7 & 475030 & 12.9 & 21.4 & Promedio & 17.47 & 19.78 \\
\hline 474521 & 14.6 & 25 & 475071 & 14.7 & 14.2 & & & \\
\hline
\end{tabular}

Tabla 4. Datos resumidos del Análisis comparativo de los métodos de microfases para el cobre. (Xuejing Xie et al. 2011)

\begin{tabular}{|l|c|c|c|}
\hline \multirow{2}{*}{\multicolumn{1}{c|}{ Método }} & \multirow{2}{*}{$\begin{array}{c}\text { Mediana } \\
\text { (ppm) }\end{array}$} & \multicolumn{2}{c|}{$\begin{array}{c}\text { No anómalo de muestras } \\
\mathbf{2 0} \text { ppm como umbral }\end{array}$} \\
\cline { 3 - 4 } & & $\begin{array}{c}\text { Encima del } \\
\text { deposito }\end{array}$ & área de background \\
\hline Muestras de malla 80 & 16.8 & 17 & 3 \\
\hline Fase oxidada & & & 2 \\
\hline Muestras Procesadas & 16.8 & 18 & \\
\hline Fase oxidada & & & 11 \\
\hline Muestras de malla 80 & 14.7 & 11 & \\
\hline Fase primaria de Sulfuro & & & \\
\hline Muestras Procesadas & 17.7 & 19 & \\
\hline Fase primaria de Sulfuro & & & \\
\hline
\end{tabular}




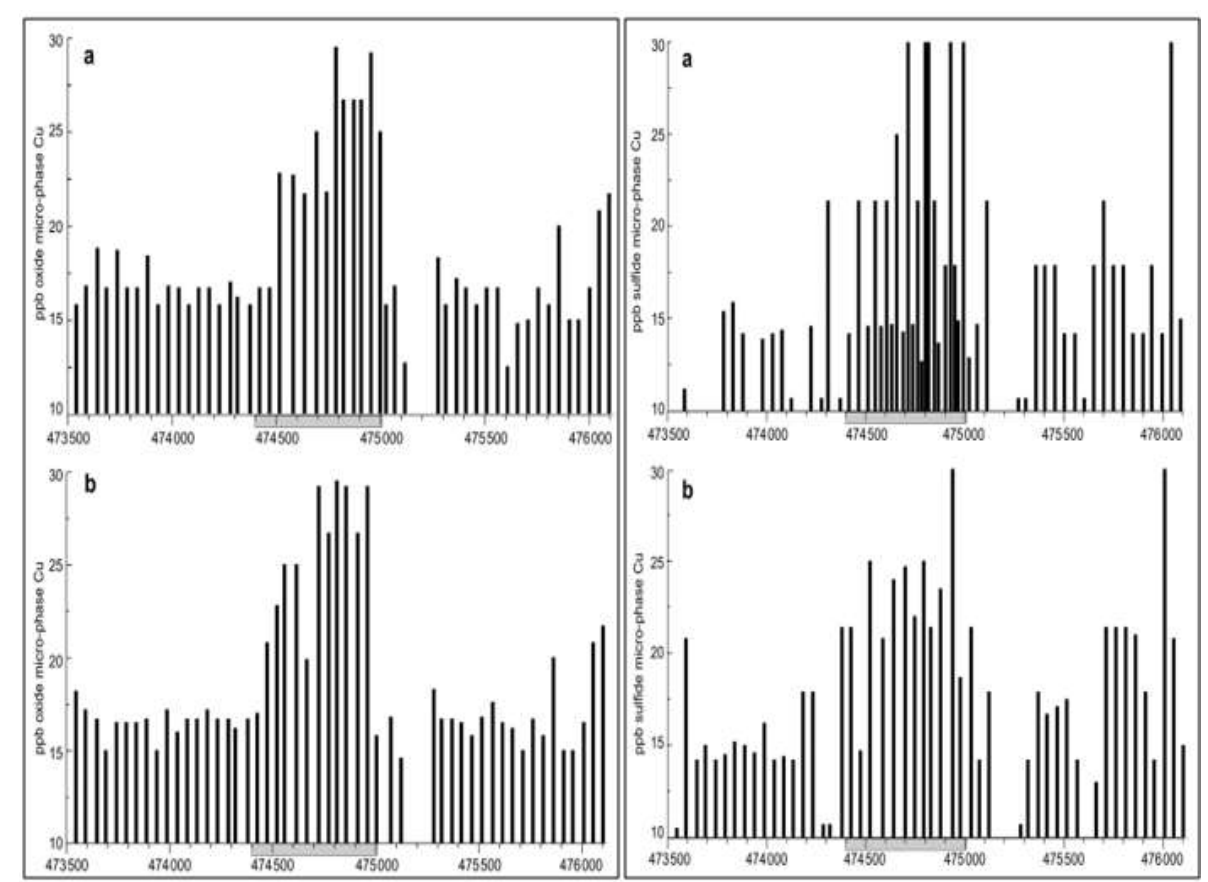

Figura 6. Diagramas de $\mathrm{Cu}$ en microfases de óxido; cuadros de la izquierda. Diagramas de $\mathrm{Cu}$ en microfases de sulfuros cuadros de la derecha; posición del cuerpo mineral oculto identificado por barra sombreada horizontal. (Xuejing Xie et al. 2011)

\subsection{Aplicaciones Isotópicas: Hidrogeoquímica isotópica en la exploración minera.}

La información sobre el entorno del subsuelo es escasa. Por lo tanto, el objetivo de aplicar sistemas isotópicos a la hidrogeoquímica es doble (1) disminuir el coste de la exploración encubierta al aumentar la huella de los yacimientos, lo que se traduce en la recogida de menos muestras, y (2) reducir el riesgo percibido de la orientación mediante la hidrogeoquímica. El fraccionamiento de los metales y su movilización como solutos disueltos en las aguas subterráneas (figura 6) proporciona una forma potencial de detección directa en el entorno secundario, además de proporcionar información contextual crucial que indica las fuentes de agua, las pruebas de mezcla y la interacción agua-sulfuro. (James Kidder, et al, 2019). En las décadas transcurridas desde que Harold Urey (White 2015) fue pionero en el uso de isótopos estables ligeros, los avances analíticos y científicos han hecho que la lista de elementos disponibles para el análisis aumente hasta incluir elementos ligeros como el Li y el B, así como elementos pesados $\mathrm{Ca}, \mathrm{Cl}, \mathrm{Cu}, \mathrm{Fe}, \mathrm{Hg}, \mathrm{Mg}$, Mo, Se, Si, U y Zn (White 2015). En última instancia, cualquier elemento con 2 o más isótopos o un isótopo radiogénicos o cosmogénicos pueden utilizarse para el rastreo isotópico (Kyser 2017). El uso de la hidrogeoquímica de las aguas subterráneas y superficiales como forma viable de detección directa en la 
exploración minera ha sido promovido durante mucho tiempo por investigadores como Boyle et al. (1971) Cameron (1978); Miller et al. (1982); Goodfellow (1983); y Runnells (1984). Los primeros trabajos se centraron en los yacimientos sedimentarios de uranio en Canadá durante los años 70 y principios de los 80 (Cowart y Osmond 1977, Deutscher et al. 1980; Dyck y Hood 1979; y Appleyard 1984), aunque en los últimos años la mayoría de los estudios se han centrado en los yacimientos de metales básicos (J. Ruijin et al. 1994; Leybourne et al. 2003; Phipps et al. 2004; Patrice de Caritat 2005; Leybourne et al. 2006).

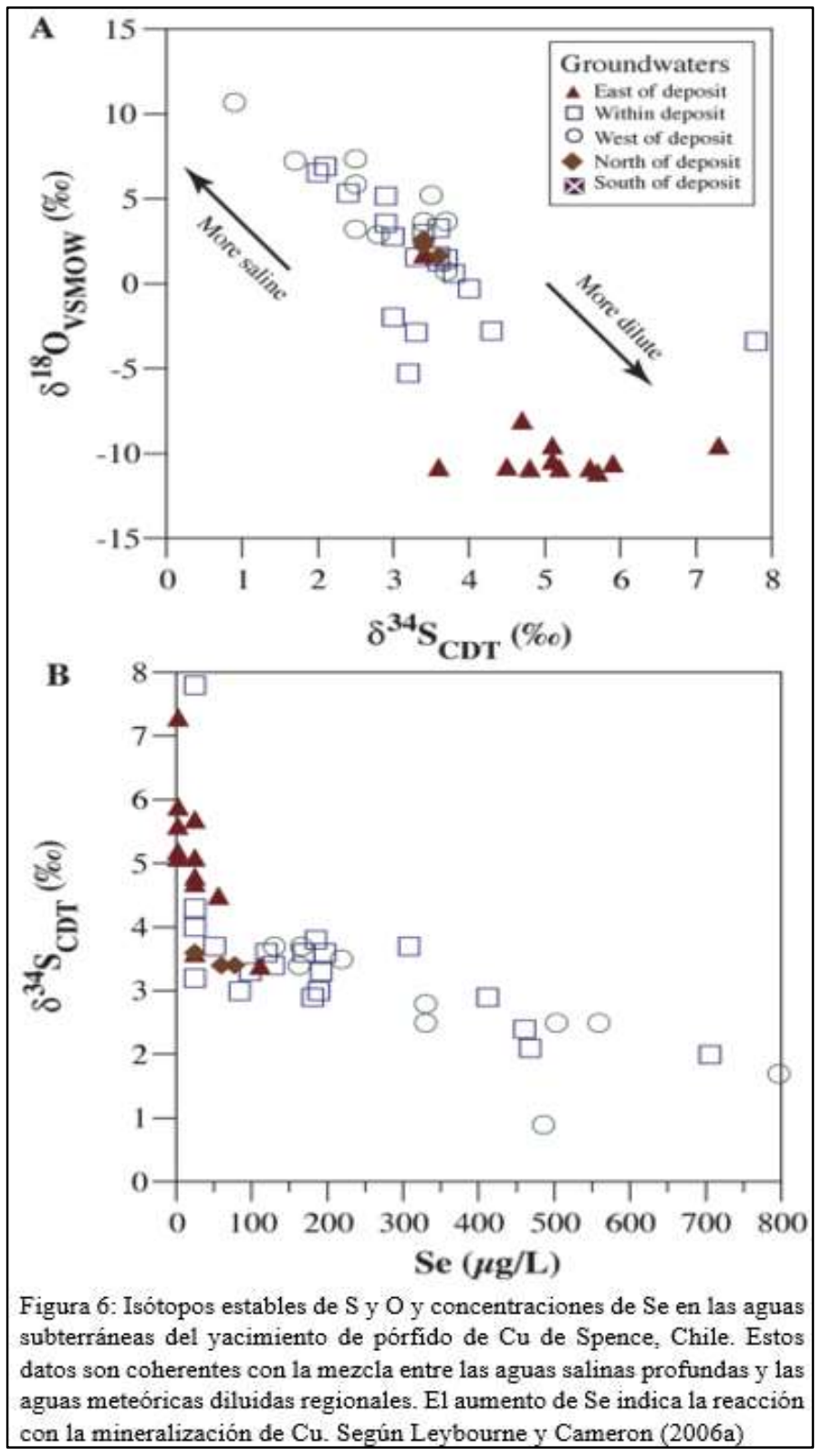


El renovado interés por la técnica se produjo tras el desarrollo y la aplicación de la espectrometría de masas con plasma acoplado inductivamente (ICP-MS), que permite el análisis de soluciones hasta límites de detección inferiores a las ppt (Stetzenbach et al. 1994; Hall et al. 1995). Esto supuso un avance fundamental, ya que anteriormente la baja solubilidad y la abundancia de los elementos contaminantes en las aguas subterráneas restringían el uso generalizado de la técnica (Runnells 1984). Ahora que el análisis del agua se ha convertido en una rutina en los laboratorios comerciales, con un plazo de entrega de muestras relativamente rápido, es posible analizar una amplia gama de elementos, lo que facilita la elaboración de modelos geoquímicos. (James Kidder, et al, 2019).

El modelo de dos etapas para el enriquecimiento supergénico en el desierto de Atacama y la datación en la serie $U$ de ensambles de yeso-atacamita muestra que los procesos de oxidación supergénica en los depósitos de $\mathrm{Cu}$ estuvieron activos durante el Pleistoceno Medio y Tardío, cuando prevalecían las condiciones climáticas hiperáridas en el desierto de Atacama. Los datos isotópicos de los minerales supergénicos definen un extenso período de oxidación supergénica entre 45 y 9-5 Ma con un pico en 21-14 Ma, dominado por la circulación descendente de agua meteórica bajo condiciones climáticas de semiáridas a áridas. Un período de transición, caracterizado por una brecha en los datos isotópicos (entre $\sim 5$ y $2 \mathrm{Ma}$ ) precede a la etapa tardía de la oxidación supergénica dominada por la formación y conservación de atacamita en condiciones de hiperaridez $\left(<1,5 \mathrm{Ma},{ }^{36} \mathrm{Cl}\right)$. Las edades absolutas de formación de la atacamita en varios yacimientos de cobre se agrupan entre $\sim 250$ y 75 ka (Pleistoceno medio a tardío), (recuadro de la derecha en figura 7).

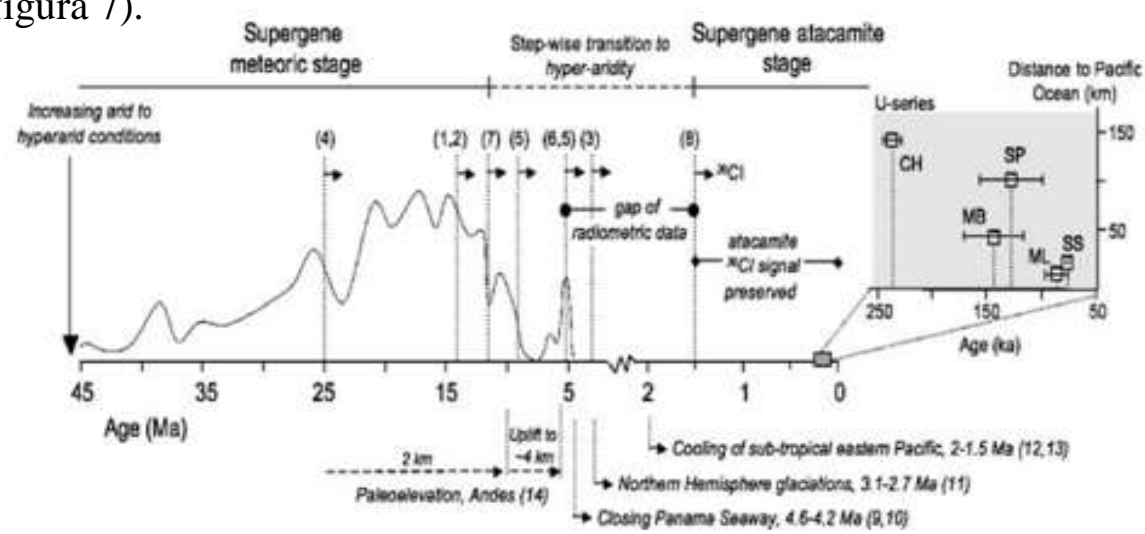

fig.7. Cronologia del enriquecimiento supergénico de los depósitos de cobre en el desierto de Atacama, Chile, y su relación con el cambio climático en la región. Las líneas verticales de puntos marcan el inicio de la hiperaridez según diferentes autores (números), con la prevalencia de la hiperaridez hacia la derecha (dirección de la flecha). Referencias: (1) Alpers and Brimhall (1988), (2) Sillitoe and McKee (1996), (3) Hartley and Chong (2002), (4) Dunai et al. (2005), (5) Arancibia et al. (2006), (6) Hartley and Rice (2005), (7) Rech et al. (2006), (8) Reich et al. (2008), (9) Haug and Tiedemann (1998), (10) Haug et al. (2001), (11) Haug et al. (1999), (12) Cannariato and Ravelo (1997), (13) Ravelo et al. (2004), (14) Garzionelet al. (2008) 


\section{DISCUSION}

- El enriquecimiento supergénico y la formación de depósitos de cobre de alta ley en el norte de Chile, ocurrieron esencialmente en periodos semiáridos, desde el Mioceno temprano a medio (Alpers y Brimhall 1988). El levantamiento de los Andes durante el Mioceno Medio creó una barrera para la penetración hacia el oeste de la humedad del Amazonas y una simultánea disminución de la evaporación desde el sur del Océano Pacífico debido al fortalecimiento de la corriente de Humboldt a lo largo de la costa de Perú-Chile condujo a la hiperaridificación del desierto de Atacama (Alpers y Brimhall 1988). Esta hiperaridificación ralentizó el proceso de enriquecimiento supergénico y la consiguiente y simultánea disminución de las tasas de erosión ayudó a preservar los mantos de enriquecimiento supergénico que se habían formado por las capas freáticas en constante descenso (Alpers y Brimhall 1988)

- Un resultado notable de la datación por desequilibrio U-Th del yeso de los intercrecimientos de yeso-atacamita, apoyado por los datos de ${ }^{36} \mathrm{Cl}$ de la atacamita, es que este mineral se formó en un ceñido intervalo de tiempo geológico; entre los 237 y $75 \mathrm{Ka}$ en toda el área examinada del norte de Chile.

- La meteorización de la mineralización de sulfuros primarios (mineralización hipógena) en estos depósitos produjo varios cientos de metros de capas de grado superior de sulfuros de $\mathrm{Cu}$ secundarios, óxidos, hidróxidos, carbonatos, sulfatos y cloruros. Esta mineralización supergénica es un reflejo de la historia del flujo de agua subterránea, la tectónica, el paisaje y el clima del desierto de Atacama (Alpers y Brimhall 1988; Sillitoe y McKee 1996; Chávez 2000; Clarke 2006).

- Los estudios confirman la ocurrencia de procesos de dispersión de iones y nanopartículas transportadas a partir de yacimientos de sulfuros bajo cobertura, con generación de improntas geoquímicas de contraste en superficie, conceptos aplicables para la prospección geológica en zonas densamente cubiertas.

- La estrecha correlación espacial entre los depósitos de $\mathrm{Cu}$ con atacamita y las principales estructuras de la región, junto con la alta salinidad y la composición isotópica de las aguas de las que precipitó (Leybourne y Cameron 2006; 2008; Reich et al. 2008) sugiere que el enriquecimiento supergénico en condiciones de hiperaridez ha sido impulsado por la circulación ascendente de aguas salinas profundas durante el fallamiento activo a lo largo de las estructuras principales abiertas o reactivadas por 
la intensa actividad sísmica (Armijo y Thiele 1990; Cameron et al. 2002; 2007; Vargas et al. 2005; González et al. 2006).

- Los argumentos sedimentológicos datan el inicio de la hiperaridificación del desierto de Atacama en torno a los 12 Ma (Jordan et al., 2014). Los resultados de Caroline Sánchez, 2017; proporcionan la primera evidencia directa de una drástica caída en la tasa de denudación hacia los 10-12 Ma, confirmando la hiperaridificación del desierto de Atacama en esta época. Los resultados sugieren que $100 \mathrm{~m}$ de sedimentos se depositaron en menos de $\sim 2$ Ma. Esta tasa de sedimentación relativamente alta $(>=50$ $\mathrm{m}$ por My) contrasta con las tasas de sedimentación y denudación inferidas para el periodo neógeno en el desierto de Atacama (Maksaev y Zentilli, 1999; Alpers y Brimhall, 1988). Una mayor erosión desde el origen y el bloqueo por la reactividad de fallas miocénicas como la del Llano o Tesoro, que alteraron la topografía con desplazamientos verticales de varias decenas de metros, pueden explicar este evento de sedimentación. Esta elevada tasa de sedimentación cesó hacia los $10 \mathrm{Ma}$, en consonancia con la hiperaridificación de esa época.

\section{CONCLUSIONES}

- El enriquecimiento supergénico es esencial para la viabilidad económica de muchos yacimientos de pórfidos de cobre (Reich y Vasconcelos 2015) y determinar cuándo y cómo se ha producido el enriquecimiento supergénico es importante a la hora de explorar yacimientos de cobre enriquecidos y exóticos (Mote et al. 2001). También al determinar qué, dónde, cómo y cuándo se producen las reacciones químicas en la corteza meteorizada, podemos descifrar las historias tectónicas y climáticas de la Tierra durante la formación de los yacimientos supergénicos. (Caroline Sánchez, 2017).

- El enriquecimiento supergénico de los depósitos de Cu en el desierto de Atacama en el norte de Chile ha sido el factor principal para que esta región se convierta en la mayor productora de $\mathrm{Cu}$ del mundo.

- La superposición de datos hidrogeoquímicos e isotópicos permite la identificación definitiva de la mineralización en aguas subterráneas. Sugerimos que la toma de muestras para el análisis isotópico sea la norma en labores de prospección minera en terrenos densamente cubiertos. (James Kidder et al, 2019). 
- El engrosamiento del perfil meteorizado y el grado de enriquecimiento en cobre depende, probablemente de un sutil equilibrio entre la tasa de precipitación y la tasa de exhumación de un pórfido de cobre, controlando ambas los tiempos de residencia del agua en las zonas vadosa y saturada (Brimhall et al., 1985). Aunque el equilibrio entre los procesos geomórficos, climáticos y tectónicos parece ideal en el desierto de Atacama para la formación de yacimientos enriquecidos en supergenes, también se dan y se conservan en otros entornos. Por lo tanto, para cuantificar las tasas de los parámetros implicados en dicho equilibrio, una primera aproximación es considerar este equilibrio como una relación entre la tasa de erosión y la tasa de precipitación. (Caroline Sánchez, 2017)
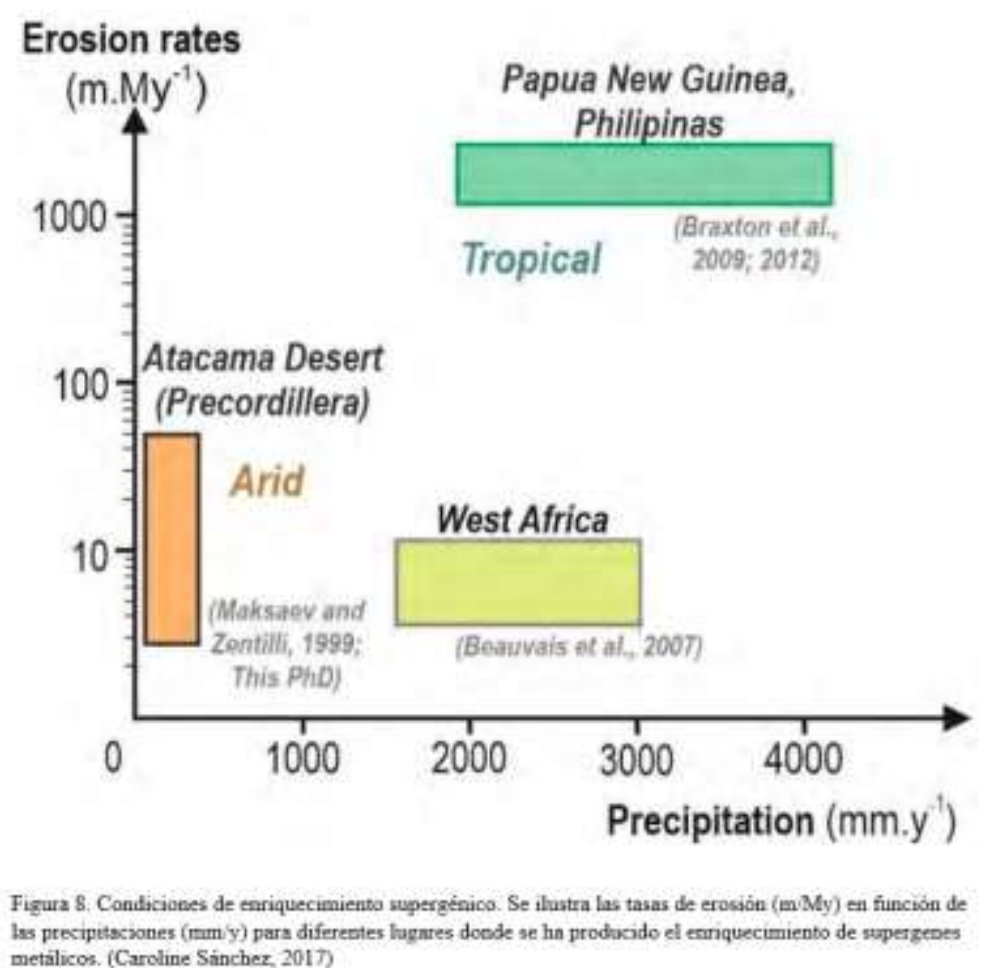

- Los métodos analíticos de microfases para el cobre que se exponen en este documento pueden usarse en exploración geoquímica o incorporarse con técnicas de lixiviación selectiva en esfuerzos detallados para aumentar la tasa de éxito en la búsqueda de depósitos de pórfido de cobre ocultos bajo terrenos desérticos. La existencia de microfases de minerales sulfurados en cubiertas exógenas puede servir como evidencia más concreta de la existencia de depósitos de minerales enterrados. (Xuejing Xie, et al. 2011). En realidad, el sulfuro de $\mathrm{Cu}$ primario en el mineral puede estar en 
nanoescala y adherirse a microburbujas de gas o vapor de agua a medida que migra hacia la superficie (Xie y Wang, 2003).

- Los métodos de microfases, pueden usarse en Prospección Geoquímica con técnicas de lixiviación selectiva para aumentar la tasa de éxito en la ubicación de pórfidos de cobre ocultos bajo terrenos desérticos y la existencia de fases de minerales oxidados y sulfurados en cubiertas exógenas sirve como evidencia concreta de la existencia de depósitos de minerales enterrados profundamente.

- Los trabajos en cuatro lugares del desierto de Atacama que se extienden hasta el norte de Chuquicamata demuestran tener el potencial de ser ampliamente aplicados en el desierto de Atacama y potencialmente en otros entornos desérticos tectónicamente activos, para el descubrimiento de depósitos de pórfidos profundamente enterrados. (Brown et al. 2019).

\section{REFERENCIAS BIBLIOGRAFICAS}

James Kínder, Matthew I. Melbourne, Alexandre Boicot, Daniel Layton-Matthews, 2020; Isotope geochemistry of groundwaters in mineral exploration - the tip of the iceberg.

Alexandra Brown, Peter A. Winterburn \& Thomas Bissig, 2019; Geochemical signature of earthquake-induced surface flooding by mineralized groundwater over the buried Atlántida Deposit, Northern Chile.

Alberto Fernández, 2019; Sedimentología del Cenozoico del Distrito Minero Centinela y de la Cuenca Quebrada El Profeta (Desierto de Atacama, Chile): evolución paleoambiental y génesis de los depósitos de $\mathrm{Cu}$-exótico

Benedikt Ritter, Finlay M. Stuart, Steven A. Binnie, Axel Gerdes, Volker Wennrich \& Tibor J. Dunai, 2018; Neogene fluvial landscape evolution in the hyperarid core of the Atacama Desert.

Luis Carvilla, 2018; Una visión moderna de la orogénesis en el ámbito de la tectónica de placas.

Brian K. Horton, 2018; Tectonic Regimes of the Central and Southern Andes: Responses to Variations in Plate Coupling During Subduction.

Caroline Sanchez, 2017; Évolution de la dénudation et contexte géomorphologique des minéralisations cuprifères supergènes dans le district Centinela, désert 
d'Atacama, Chili, à partir de la thermochronologie et des nucléides cosmogéniques.

Rodrigo Riquelme, 2017; Supergene and exotic $\mathrm{Cu}$ mineralization occur during periods of landscape stability in the Centinela Mining District, Atacama Desert

Paulo M. Vasconcelos, Martin Reich, and David L. Shuster; 2015; The Paleoclimatic Signatures of Supergene Metal Deposits.

Martin Reich, 2015; Geochemical and paleoclimatic signatures of supergene metal deposits.

Leybourne, M.I. and Cameron, E.M., 2015; Groundwater's in Geochemical Exploration: Methods, Applications, and Future Directions.

Martin Reich, Glen T. Snyder, Fernanda Álvarez, Alida Pérez, Carlos Palacios, Gabriel Vargas, Eion M. Cameron, Yasuyuki Muramatsu and Udo Fehn, 2013; Using Iodine isotopes to constrain supergene fluid sources in arid regions: insights from the chuquicamata oxide blanket

Xuejing Xie, Yinxiu Lu, Wensheng Yao, Jinfeng Bai, 2011; Further study on deep penetrating geochemistry over the Spence porphyry copper deposit, Chile.

Carlos Palacios \& Olivier Rouxel \& Martin Reich \& Eion M. Cameron \& Matthew I. Leybourne, 2010; Pleistocene recycling of copper at a porphyry system, Atacama Desert, Chile: $\mathrm{Cu}$ isotope evidence.

Eion M. Cameron, Matthew I. Leybourne, Martin Reich \& Carlos Palacios, 2010; Geochemical anomalies in northern Chile as a surface expression of the extended supergene metallogenesis of buried copper deposits.

Eion M. Cameron1, Matthew I. Leybourne, Martin Reich \& Carlos Palacios, 2010; Geochemical anomalies in northern Chile as a surface expression of the extended supergene metallogenesis of buried copper deposits

Luis Moreno, Alejandro Sanhueza, Juan Fajardo, Reinaldo Guzmán, Marlene Lagos, Marcelo Nava, Emilio González, Alberto Ruggiero, 2009; Mineralización Hipógena del Pórfido Paleoceno Spence.

M.F. Aspadiar; R.R. Anand and Gray, 2008; Geochemical dispersion mechanisms through transported cover: implications for mineral exploration in Australia.

Martin Reich \& Carlos Palacios \& Gabriel Vargas \& Shangde Luo \& Eion M. Cameron \& Matthew I. Leybourne \& Miguel A. Parada \& Alejandro Zúñiga \& Cheng-Feng 
You, 2008; Supergene enrichment of copper deposits since the onset of modern hyperaridity in the Atacama Desert, Chile.

Peter L. Nester, Eugenia Gayo, Claudio Latorre, Teresa E. Jordan, and Nicola's Blanco, 2007; Perennial stream discharge in the hyperarid Atacama Desert of northern Chile during the latest Pleistocene.

Jason A. Rech Brian S. Currie, Greg Michalski, Angela M. Cowan, 2006; Neogene climate change and uplift in the Atacama Desert, Chile.

Eion M. Cameron \& Matthew I. Leybourne, 2005; Relationship between groundwater chemistry and soil geochemical anomalies at the Spence copper porphyry deposit, Chile.

Cameron, Eion \& Hamilton, Stewart \& Leybourne, Matthew \& Hall, G. \& Mc Clenaghan, M.B., 2004; Finding deeply buried deposits using geochemistry.

Cameron, Eion \& Leybourne, Matthew \& Kelley, David, 2002; Exploring for deeply covered mineral deposits: Formation of geochemical anomalies in northern Chile by earthquake-induced surface flooding of mineralized groundwater.

Alpers CN, Brimhall GH (1988) Middle Miocene climatic change in the Atacama Desert, northern Chile: evidence from supergene mineralization at La Escondida. Geol Soc Am Bull 100:1640-1656. 